\title{
Urgences
}

\section{Les trahisons de l'hyperbole}

\section{Marie Bélisle}

Numéro 21, novembre 1988

Bagatelles et crases

URI : https://id.erudit.org/iderudit/025491ar

DOI : https://doi.org/10.7202/025491ar

Aller au sommaire du numéro

Éditeur(s)

Urgences

ISSN

0226-9554 (imprimé)

1927-3924 (numérique)

Découvrir la revue

Citer ce document

Bélisle, M. (1988). Les trahisons de l'hyperbole. Urgences, (21), 9-18.

https://doi.org/10.7202/025491ar

Ce document est protégé par la loi sur le droit d'auteur. L'utilisation des services d'Érudit (y compris la reproduction) est assujettie à sa politique d'utilisation que vous pouvez consulter en ligne.

https://apropos.erudit.org/fr/usagers/politique-dutilisation/
Cet article est diffusé et préservé par Érudit.

Érudit est un consortium interuniversitaire sans but lucratif composé de l’Université de Montréal, l'Université Laval et l'Université du Québec à Montréal. Il a pour mission la promotion et la valorisation de la recherche. https://www.erudit.org/fr/ 


\title{
MARIE BELISLE \\ Les trahisons de l'hyperbole
}

\author{
1. \\ le jeu des nombres ment comme une fuite hasardeuse et lente \\ .2. \\ hors du genre la règle des brisures \\ désormais masque l'appel sexué \\ .3. \\ insinué gardé dans les replis et \\ dans les failles des robes odorantes \\ saisi comme le discret plaisir de
}

1. ou comme 10cret plaisir. «Il y a de l'écriture dans l'équation.» (Michel Cassé, «Épreuves d'écriture», Les immatériaux, Paris, Éditions du Centre Georges Pompidou, 1985, p. 55)

2. Langage: «Espèce d'écriture! Langue, lèvre (en hébreu), appel.» (Jacques Derrida, id., p. 111) 


\section{QUELQUES REGLES DU GENRE}

Le jeu des règles ment.

«Le jeu n'est pas seulement le divertissement: c'est aussi la règle.» (Jacques Bens, «Écrire Aujourd'hui, autoportraits d'écrivains sur fond de siècle», Autrement, Paris, no. 69, 1985, p.48)

Que l'on s'entende bien: la lecture est un déchiffrement. «Écriture (défrichage) devient écrit. L'écrit s'évaporant au fur de la lecture (déchiffrage) se condense en récit.» (Maurice Roche, «Épreuves d'écriture», Les immatériaux, op. cit., p. 56)

«L'écriture ment de naissance. [...] La première page connaît la dernière; toute ligne est écrite après chacune des autres.» (Paul Caro, id., p. 55)

«Comment saurai-je que le (texte) est fini? En somme, comme toujours, il s'agit d'élaborer une langue. Or dans toute langue les signes reviennent et, à force de revenir, ils finissent par saturer le lexique l'oeuvre.» (Roland Barthes, Roland Barthes par Roland Barthes, Paris, Seuil, coll. «Écrivains de toujours», 1976, p. 166)

«Nous le savons, lire, c'est explorer les relations spécifiques par lesquelles sont liés les éléments d'un texte.» (Jean Ricardou, Le nouveau roman, Paris, Seuil, coll. «Écrivains de toujours», 1978, p. 70)

«Le texte est une productivité. [...] même écrit (fixé) il n'arrête pas de travailler, d'entretenir un processus de production.» (Roland Barthes, «Texte (théorie du)», Encyclopedia Universalis, vol. 15, Paris, 1980, p. 1015) 
l'offre comme le chant du verre sous le doigt mouillé juste avant

la cassure quand persiste

le sens du toucher sur la langue

.5 .

là où s'établit la certitude

du déplacement des codes la striction des tissus confondus comme les corps

et les mots couchés analogues on the sheet

à la minute même du frémissement et de l'apnée

\section{6 .}

le signe de la voix le gémissement bref immiscé étroitement dans la fibre soudain épidermique s'accroît le mouvement de la nuque trace des graphes dérivés livrés

au jeu des aimants nous nous perdons dans l'écart magnifique des angles la figure de

4. Langage: «Dans son sens étymologique, tentative d'approche sexuelle par l'intermédiaire des muqueuses de la langue.» (Philippe Curval, "Épreuves d'écriture», Les immatériawx, op. cit., p. 112) 


\section{APPROXIMATIONS}

En apparence, dix petits textes numérotés, comportant chacun le nombre de vers correspondant à leur emplacement dans la série, l'ordinal déterminant le cardinal. Au total, donc, cinquante-cinq vers.

En fait, un seul long texte à charnières: dix intersections ont été «cachées» dans les textes et chacune d'elles ouvre quatre voies. Il y aurait donc, théoriquement, $410(1,048,576)$ chemins menant du premier au dernier mot. Toutefois, quelques avenues sont bloquées et certaines intersections n'ouvrent qu'une ou deux voies; ce qui laisse, tout de même, au moins 50,000 voies ouvertes! Par ailleurs, puisque plusieurs intersections permettent de remonter le cours du texte, il est possible d'emprunter successivement plusieurs voies sans jamais se résoudre au glissement final, ce «signal du recommencement» posé au cinquante-cinquième vers. 
la frontière où s'amorce enfin l'approche nous déportant d'une année sur l'autre toujours neufs par la constante du fleuve entre nos cuisses et par la venue du rouge aux lèvres encore plus vive quand l'intervalle pose une fois pour toutes la $f(r)$ iction le désir

.8 .

en telle circonstance atténuée par l'urgence curieusement calculée je sais bien que les chambres d'hôtel ne suffisent pas les prétextes manquent le téléphone nous trahit les rendez-vous nous échappent je doute de toute tentative de préméditation même lorsqu'à travers la peau du coeur comme en une dérive vers quelque lieu de bagatelle obscur(e) 


\section{ATTENTION}

Le parcours n'est pas linéaire; les intersections n'apparaissent pas dans l'ordre. Exemple: le renvoi au texte 5. survient dans le texte 9.

La distribution des intersections dans les textes est irrégulière; certains textes peuvent n'en comporter aucune, d'autres en cacher plusieurs.

La structure est récursive. Selon les voies choisies, la relecture d'un passage peut être nécessaire pour atteindre l'intersection suivante.

La structure syntaxique est mouvante; la relecture d'un passage peut impliquer un redécoupage du texte et un renversement du sens.

La ponctuation, absente dans les textes peut être posée à la lecture; ainsi, les textes en vers peuvent se transformer en un seul texte en prose dont la ponctuation dépend des voies choisies (la section Exploration en constitue un exemple). 


$$
.9 .
$$

nous ne nous dérobons plus je sais qu'alors nous cédons à l'odeur appréhendée aux remous prévisibles au goût de la salive alors parfois presque malgré tous les battements s'accélèrent alors se synchronisent sous les vêtements les sueurs s'écrasent par effet de résistance la tension se fixe dans les capillaires ta main se pose là nous ne sourions plus graves dans le 


\section{INTERSECTIONS}

Donc, dix charnières cachées: numériques bien sûr. Le lecteur doit être prêt à les «entendre» au fil des textes:

commun (1), hasardeuse (2), étroitement (3), lorsqu'à travers (4), synchronisent (5), persiste (6), s'établit (7), fuite (8), neufs (9), discret (10).

À l'intersection, quatre voies ouvertes:

1. la ligne droite: faire comme si on n'avait rien entendu et poursuivre la lecture en empruntant l'avenue principale, celle de la succession linéaire des mots et des vers. Ex: Le jeu des nombres ment comme une fuite hasardeuse et lente...

2. le nième texte: se rendre au texte correspondant au chiffre identifié et l'aborder par la voie habituelle, le premier mot. Exemple: Le jeu des nombres ment comme une fuite... (texte 1) en telle circonstance atténuée... (début du texte 8)

3. la nième ligne du nième texte: se rendre illico à la dernière ligne du texte correspondant au chiffre identifié. Exemple: «le jeu des nombres ment comme une fuite [...]» (texte 1) «une dérive vers quelque lieu de bagatelle obscur(e)» (8ème ligne du texte 8)

4. le nième mot du nième texte: se rendre au mot dont l'emplacement dans le texte correspond au chiffre identifié. Exemple: «le jeu des nombres ment comme une fuite [...]» (texte 1) «curieusement calculce [...]» (8ème mot et suivants du texte 8 )

Des voies supplémentaires sont parfois ouvertes. Exemple: «[...] quand persiste» (texte 4) «la figure de [...]» (6ème mot et suivants de la 6ème ligne du texte 6 ) 
frôlement des surfaces maintenant ralenti comme stoppant soudain le surgissement de l'onde dans le sang maintenus en état d'attraction nous prolongeons le vacillement jusqu'à ce que la verticale bascule par induction nous tombons sous le sens reclus en quelque lieu commun mâle et femelle dans les règles du genre glissements frottements spasmes et black-out dès lors semblablement morts jusqu'au signal du recommencement

10. Pourquoi vouloir, à tout prix, aller derrière la façade, surtout si elle est belle? les effets de façade sont comme les effets de surface: ils valent par euxmêmes, sauf pour ceux qui cherchent la triste "paix des profondeurs» (François Châtelet, «Épreuves d'écriture», Les immatériaux, op. cit., p. 71) 


\section{EXPLORATION}

Le jeu des nombres ment comme une fuite hasardeuse, masque l'appel sexué, insinué, gardé dans les replis et dans les failles des robes odorantes, saisi comme le discret surgissement de l'onde dans le sang. Maintenus en état d'attraction, nous prolongeons le vacillement jusqu'à ce que la verticale bascule; par induction nous tombons sous le sens, reclus en quelque lieu commun. Le jeu des nombres ment comme une fuite, une dérive vers quelque lieu de bagatelle obscur(e). Nous ne nous dérobons plus; je sais qu'alors nous cédons à l'odeur appréhendée, aux remous prévisibles, au goût de la salive; alors parfois, presque malgré tout, les battements s'accélèrent, se synchronisent, là où s'établit une fois pour toutes la $\mathrm{f}(\mathrm{r})$ iction, le désir. En telle circonstance, atténuée par l'urgence curieusement calculée, je sais bien que les chambres d'hôtel ne suffisent pas; les prétextes manquent, le téléphone nous trahit, les rendez-vous nous échappent. Je doute de toute tentative de préméditation, même lorsqu'à travers le chant du verre sous le doigt mouillé, juste avant la cassure, quand persiste l'écart magnifique des angles, la figure de la frontière (où s'amorce enfin l'approche) nous déportant d'une année sur l'autre, toujours neufs, nous ne sourions plus. Graves dans le frôlement des surfaces maintenant ralenti, comme stoppant soudain le surgissement de l'onde dans le sang, maintenus en état d'attraction, nous prolongeons le vacillement jusqu'à ce que la verticale bascule par induction. Nous tombons sous le sens, reclus en quelque lieu commun, mâle et femmelle dans les règles du genre: glissements, frottements, spasmes et black-out, dès lors semblablement morts jusqu'au signal du recommencement. 